\title{
EFEITO DO FENO DE FLOR-DE-SEDA SOBRE A CARCAÇA E CONSTITUINTES CORPORAIS DE CORDEIROS MORADANOVA
}

\author{
EFFECT OF SILK FLOWER HAY ON CARCASS AND NON CARCASS COMPONENTS \\ THE MORADANOVALAMBS
}

Silva, N.V. da ${ }^{1 *}$, Costa, R.G. ${ }^{2}$, Medeiros, A.N. $\mathrm{de}^{2}$, Azevedo, P.S. $\mathrm{de}^{2}$, Carvalho, F.F.R. $\mathrm{de}^{3}$, Medeiros, G.R. de ${ }^{4}$ e Madruga, M.S. ${ }^{5}$

\begin{abstract}
1'Departamento de Agropecuária. Instituto Federal de Pernambuco (IFPE). São Pedro. Belo Jardim, PE. Brasil. *nelson.silva@belojardim.ifpe.edu.br

${ }^{2}$ Departamento de Zootecnia. Universidade Federal da Paraíba (CCA/UFPB). Areia, PB. Brasil. betogermano@hotmail.com

${ }^{3}$ Departamento de Zootecnia. Universidade Federal Rural de Pernambuco (UFRPE). Recife, PE. Brasil. ${ }^{4}$ Instituto Nacional do Semiárido (INSA). Campina Grande. PB. Brasil.

${ }^{5}$ Departamento de Tecnologia e Química de Alimentos. Universidade Federal da Paraíba (UFPB/DTQA). João Pessoa, PB. Brasil.
\end{abstract}

\section{PalaVRas chaVe ADICIONAIS \\ Calotropis procera. Carne. Gordura.}

\section{RESUMO}

Avaliou-se a utilização do feno de flor-deseda (Calotropis procera SW) (FFS) em cordeiros Morada Nova em confinamento. Utilizaram-se 32 cordeiros com peso vivo inicial de 12,72 $\pm 1,99 \mathrm{~kg}$ e de abate $19,29 \pm 2,25 \mathrm{~kg}$, distribuídos em delineamento inteiramente casualizado com 4 tratamentos $(0 \%, 15 \%, 30 \%$ e $45 \%$ de FFS) e oito repetições. $\mathrm{O}$ aumento dos níveis de FFS diminuiu a conformação da carcaça $(2,31 ; 2,58 ; 2,17 ; 1,81$ pontos), perímetro da coxa $(33,38 ; 32,17 ; 32,17$; $30,00 \mathrm{~cm})$, compacidade da carcaça $(0,18 ; 0,18$ $0,17 ; 0,15 \mathrm{~cm})$, meia carcaça reconstituída $(4,52$; $4,63 ; 4,43 ; 3,69 \mathrm{~kg})$, lombo $(0,66 ; 0,69 ; 0,64 ; 0,50$ $\mathrm{kg})$, paleta $(0,83 ; 0,86 ; 0,80 ; 0,67 \mathrm{~kg})$ e costela $(1,13 ; 1,09 ; 1,05 ; 0,84 \mathrm{~kg})$, mas aumentou o linearmente os pesos da perna $(30,98 ; 30,81$; $31,60 ; 34,47 \mathrm{~kg})$ e a gordura mesentérica $(1,02$; $0,65 ; 1,17 ; 1,42 \%)$. A inclusão de FFS na dieta promoveu efeito sobre as medidas da carcaça e seus rendimentos, assim como o rendimento dos cortes comerciais e componentes não-constituintes da carcaça. Estima-se que a inclusão de até $30 \%$ de FFS na dieta é relevante na manutenção das boas características de carcaça em regiões onde se tenha oferta desta forragem.

\author{
AdditionAl KEYWORDS \\ Calotropis procera. Fat. Meat.
}

\section{SUMMARY}

The objective of this study was to evaluate the use of silk flower (Calotropis procera SW) hay in 32 castrate male lambs of Morada Nova breed, in feedlot, fed with diets containing 0.0, 15.0, 30.0, and $45.0 \%$ of silk flower hay (SFH). The animals $(12.72 \pm 1.99 \mathrm{~kg} \mathrm{BW})$ were slaughtered at $19.29 \pm 2.25 \mathrm{~kg}$ BW. A completely randomized design, with four diets and eigth replicates, was used. Increasing SFH levels reduced carcass conformation $(2.31,2.58,2.17$ and 1.81 points), leg perimeter $(33.38,32.17,32.17,30.00 \mathrm{~cm})$, carcass compacity (live weigth/carcass length) $(0.18,0.18$, $0.17,0.15 \mathrm{~cm}$ ), corrected half carcass weight $(4.52,4.63,4.43,3.69 \mathrm{~kg})$, loin weight $(0.66,0.69$, $0.64,0.50 \mathrm{~kg})$, shoulder weight $(0.83,0.86,0.80$, $0.67 \mathrm{~kg})$ and rib weight $(1.13,1.09,1.05,0.84 \mathrm{~kg})$, while there was a linear increase in leg weight $(30.98,30.81,31.60,34.47 \mathrm{~kg})$ and mesenteric fat percentage $(1.02,0.65,1.17,1.42 \%)$. Increasing dietary levels of SFH to lambs affected carcass measurements and carcass yield, retail cuts and non-carcass components. It is thought that the inclusion of $30 \%$ of SFH in the diet is excellent for the maintenance of carcass characteristics in regions where there is access to this fodder plant. 


\section{INTRODUÇÃO}

A flor-de-seda (Calotropis procera $\mathrm{SW}$ ) tem um elevado teor de proteína e alta digestibilidade, aliado a resistência ao baixo índice pluviométrico, o que a caracteriza como uma alternativa eficaz na suplementação de proteínas e carboidratos para pequenos ruminantes (Marques et al., 2008).

A raça Morada Nova é uma raça promissora na região Nordeste, destacando-se por sua resistência às condições climáticas, assegurando-se boa reprodução neste ambiente. Portanto, constitui-se um recurso genético fundamental à redução da pobreza e a fixação do homem no campo (Silva et al., 2007). Estudos comparativos dos aspectos morfológicos da carcaça permitem comparações entre tipos raciais, pesos e sistemas de alimentação (Osório et al., 1998) e possibilitam a caracterização de grupos genéticos quanto ao porte e aptidões. Os parâmetros quantitativos relacionados à carcaça é critério para melhorar a interpretação e avaliação do desempenho animal (Alves et al., 2003).

Os componentes não-constituintes da carcaça têm importância na dieta de algumas regiões do mundo. Por essa razão, a comercialização destes produtos pode agregar valor à atividade, constituindo até $30 \%$ do valor do animal (Santos et al., 2008).

Face às considerações, objetivou-se avaliar as características da carcaça, bem como a composição regional e o rendimento dos órgãos não-constituintes da carcaça de cordeiros da raça Morada Nova terminados em confinamento recebendo dietas com níveis crescentes de feno de flor-de-seda.

\section{MATERIALE MÉTODOS}

O experimento foi desenvolvido na Empresa de Pesquisa Agropecuária do Rio Grande do Norte(EMPARN). Utilizou-se 32 cordeiros da raça Morada Nova com peso vivo inicial de $12,72 \pm 1,99 \mathrm{~kg}$ com aproximadamente cinco meses de idade, submetidos a um período experimental de 75 dias, incluindo 15 dias de adaptação. Os animais foram confinados e abatidos, com peso médio de 19,29 $\pm 2,25 \mathrm{~kg}$. Durante o confinamento, os animais receberam dietas ad libitum na forma de ração completa, de acordo com as recomendações do NRC (1985), oferecidas duas vezes ao dia, de modo a proporcionar sobras de aproximadamente $10 \%$. Os tratamentos consistiram na substituição do milho e soja pelo feno de flor-de-seda em níveis crescentes $0 \%, 15 \%$; $30 \%$ e $45 \%$ (tabela I).

Terminado o período de confinamento, os animais foram submetidos a um jejum por 18 horas e depois foram pesados para obtenção do peso vivo ao abate (PVA). Após o abate, esfola e evisceração, obtiveram-se o peso da carcaça quente (PCQ) e os pesos dos componentes não-constituintes da carcaça. O trato gastrintestinal (TGI) foi pesado cheio (TGIC) e em seguida esvaziado (TGIV) para obtenção do peso do corpo vazio (PCV), que foi obtido pela diferença (PCV=PVA- conteúdo do TGI). O rendimento biológico $(\mathrm{RB})$ foi obtido pela fórmula $\mathrm{RB}=(\mathrm{PCQ} / \mathrm{PCV}) \times 100$, conforme Cezar e Souza (2007).

Após refrigeração em câmara fria a $4^{\circ} \mathrm{C}$ por 24 horas, foi obtido o peso de carcaça fria (PCF). Foi calculado o rendimento de carcaça quente $(\mathrm{RCQ}=\mathrm{PCQ} / \mathrm{PVA} \times 100)$, rendimento de carcaça fria $(\mathrm{RCF}=\mathrm{PCF} / \mathrm{PVA}$ $\mathrm{x} 100)$ e a perda por resfriamento $[\mathrm{PPR}=$ (PCQ-PCF/PCQ) x 100]. Com as carcaças suspensas obtiveram-se as medidas morfométricas e o índice de compacidade da carcaça $(\mathrm{ICC}=\mathrm{PCF} /$ Comprimento interno da carcaça). No músculo Longissimus dorsi determinou-se a espessura de gordura subcutânea e a largura e profundidade máxima para estimativa do cálculo da área de olho de lombo (AOL) (De Boer et al., 1974):

$$
\left.\operatorname{AOL}\left(\mathrm{cm}^{2}\right)=(\mathrm{A} / 2 \times \mathrm{B} / 2) \times \pi\right)
$$

onde:

$A=$ distância máxima no sentido médiolateral; $\mathrm{B}=$ distância máxima no sentido dorso-ventral. 


\section{CORDEIROS MORADA NOVA ALIMENTADOS COM FENO DE FLOR-DE-SEDA}

Na meia carcaça esquerda foram realizados os cortes comerciais: pescoço (as sete vértebras cervicais, onde foi separado da cabeça, por corte oblíquo, em sua extremidade inferior entre a sétima vértebra cervical e a primeira torácica); Paleta (região que compreende a escápula, úmero, rádio, ulna e os carpos; é obtida por intermédio da secção da região axilar, através do corte dos tecidos que unem a escápula e o úmero à região torácica da carcaça); Costelas (as 13 vértebras torácicas, com as costelas correspondentes e o esterno e são obtidos através de dois cortes: o primeiro, entre a última vértebra cervical e a primeira torácica, e o segundo, entre a última vértebra torácica e a última lombar); Lombo (região das vértebras lombares, obtido perpendicularmente à coluna, entre a $13^{\mathrm{a}}$ vértebra torácica primeira lombar e última lombar -primeira sacral). Perna (cuja base óssea abrange a região do ilíaco (ílio), ísquio, púbis, vértebras sacrais, as duas primeiras vértebras coccígenas, fêmur, tíbia e tarso, é obtida por corte perpendicular à coluna entre a última vértebra lombar e a primeira sacra); Filé (é o corte especial da ponta caudal do contrafilé localizado na extensão do músculo Longissimus dorsi). Devido à perda de peso durante o processo de obtenção dos cortes através da evaporação e exsudação que as peças passam durante o retalhamento, somaram-se o peso de todos os cortes da meia-carcaça para obtenção do peso da meiacarcaça reconstituída. Com base na meiacarcaça reconstituída, obtiveram-se o peso relativo dos cortes [Corte $(\%)=($ Peso do corte/peso da meia carcaça reconstituída) $\mathrm{x}$ 100].

Para as avaliações das variáveis estudadas o delineamento experimental utilizado foi o inteiramente ao acaso, com 4 tratamentos e 8 repetições. Os dados foram submetidos à análise de variância e regressão utilizando-se o PROC GLM do SAS (1996).

Tabela I. Composição percentual e bromatológica (\%) das dietas experimentais com base na matéria seca. (Composition (\%) and bromatologic aspects of diets, on a dry matter basis).

\begin{tabular}{lcccc}
\hline & \multicolumn{4}{c}{ Níveis de feno de flor-de-seda (\%) } \\
& 0 & 15 & 30 & 45 \\
\hline Ingredientes (\%) & & & & \\
Feno de flor-de-seda & 0,00 & 9,00 & 18,00 & 27,00 \\
Feno de capim elefante & 40,00 & 40,00 & 40,00 & 40,00 \\
Milho triturado & 37,47 & 29,46 & 21,47 & 13,45 \\
Farelo de soja & 19,53 & 18,54 & 17,53 & 16,55 \\
Torta de algodão & 3,00 & 3,00 & 3,00 & 3,00 \\
& & & & \\
Composição bromatológica. Base MS (\%) & & & & \\
Matéria seca & 89,5 & 89,00 & 88,73 & 88,91 \\
Proteína bruta & 16,5 & 16,4 & 16,3 & 16,5 \\
Energia metabolizável & 2,7 & 2,7 & 2,5 & 2,2 \\
Extrato etéreo & 4,95 & 5,03 & 5,01 & 4,87 \\
Material mineral & 3,64 & 5,62 & 7,38 & 10,80 \\
Matéria organica & 96,35 & 94,37 & 92,26 & 89,20 \\
Fibra em detergente neutro & 37,9 & 40,30 & 41,13 & 46,4 \\
Fibra em detergente ácido & 5,22 & 10,46 & 12,58 & 15,08 \\
Carboidratos totais & 74,91 & 71,80 & 71,11 & 67,83 \\
Carboidratos não fibrosos & 36,9 & 31,5 & 29,98 & 21,40 \\
& & & \\
\hline
\end{tabular}




\section{SILVA, COSTA, MEDEIROS, AZEVEDO, CARVALHO, MEDEIROS E MADRUGA}

\section{RESULTADOSEDISCUSSÃO}

Na tabela II se observa que não houve efeito de regressão $(p>0,05)$ para peso vivo inicial, indicando a homogeneidade dos animais no inicio do experimento. Os consumos de matéria seca (CMS) expressos em gramas/animal/dia, $\% \mathrm{PV}$ e g/ $\mathrm{kg}^{0,75}$, variaram entre 625,1 e 739,$0 ; 3,17$ e 3,$24 ; 71,67$ e 68,67 , respectivamente, quando os níveis de substituição variaram de 0 a $45 \%$ (resultados não mostrados). Foi observada uma redução de $16 \%$ no consumo entre o menor e o maior nível de substituição, podendo ser explicado pelo aumento nos teores de FDN da dieta com a inclusão do FFS.

Houve efeito quadrático $(p<0,01)$ com a inclusão de FFS na dieta para o peso vivo ao abate. Portanto, o aumento dos níveis de FFS, os quais diminuíram os teores de energia metabolizável das dietas, além de outros nutrientes, ou o aumento nos teores de fibra em detergente neutro e fibra em detergente ácido, que pode ter causado diminuição nos teores de proteína disponíveis, influenciaram o desenvolvimento e a deposição tissular a partir de 15,75\% de FFS. Marques et al. (2007) também verificaram que os ovinos alimentados com $0,33,66$ e $100 \%$ de FFS apresentaram menores pesos de abate

Tabela II. Medidas morfométricas e características de carcaça de cordeiros da raça Morada Nova, em função dos níveis de feno de flor-de-seda (FFS) na dieta. (Morphometric measurements and carcass characteristics of lambs fed diets containing different levels of silk flower hay (SFH)).

\begin{tabular}{|c|c|c|c|c|c|c|c|}
\hline & \multicolumn{4}{|c|}{ Níveis de flor-de-seda (\%) } & \multirow[b]{2}{*}{$\mathrm{CV} \%$} & \multirow[b]{2}{*}{ Equação de regressão } & \multirow[b]{2}{*}{$\mathrm{R}^{2}$} \\
\hline & 0 & 15 & 30 & 45 & & & \\
\hline \multicolumn{8}{|l|}{ Medidas morfométricas } \\
\hline Peso inicial (kg) & 12,78 & 12,54 & 12,84 & 12,76 & 3,96 & $Y=12,72$ & - \\
\hline Peso vivo ao abate $(\mathrm{kg})^{1}$ & 19,40 & 19,93 & 19,55 & 16,23 & 9,82 & $Y=19,29+0,126 x-0,004 x^{2}$ & 0,97 \\
\hline Conformação (1-5 pts) ${ }^{1}$ & 2,31 & 2,58 & 2,17 & 1,81 & 17,17 & $Y=2,504-0,012 x$ & 0,59 \\
\hline Comprimento carcaça $(\mathrm{cm})$ & 50,63 & 51,00 & 50,50 & 49,38 & 3,79 & $Y=50,37$ & - \\
\hline Comprimento int. carcaça (cm) & 50,25 & 50,17 & 50,67 & 47,75 & 4,73 & $Y=49,70$ & - \\
\hline Comprimento perna $(\mathrm{cm})^{2}$ & 31,63 & 31,83 & 31,83 & 30,38 & 3,86 & $Y=31,56+0,057 x-0,001 x^{2}$ & 0,94 \\
\hline Perímetro coxa $(\mathrm{cm})^{2}$ & 33,38 & 32,17 & 32,17 & 30,00 & 9,91 & $Y=33,45-0,067 x$ & 0,86 \\
\hline Perímetro garupa (cm) & 45,50 & 49,83 & 48,50 & 46,75 & 13,52 & $Y=47,64$ & - \\
\hline Largura garupa $(\mathrm{cm})$ & 15,00 & 14,58 & 14,25 & 14,06 & 9,20 & $Y=14,47$ & - \\
\hline Profundidade tórax (cm) & 23,38 & 22,83 & 22,67 & 22,44 & 6,20 & $Y=22,82$ & - \\
\hline Perímetro tórax $(\mathrm{cm})$ & 61,88 & 62,00 & 62,00 & 58,63 & 5,29 & $Y=61,12$ & - \\
\hline Compacidade carcaça $(\mathrm{kg} / \mathrm{cm})^{1}$ & 0,18 & 0,18 & 0,17 & 0,15 & 9,17 & $Y=0,185-0,00001 x$ & 0,83 \\
\hline \multicolumn{8}{|l|}{ Características de carcaça } \\
\hline Peso corpo vazio $(\mathrm{kg})^{1}$ & 16,87 & 17,35 & 17,03 & 13,83 & 10,45 & $Y=16,76+0,121 x-0,004 x^{2}$ & 0,97 \\
\hline Peso carcaça quente ${ }^{2}$ & 9,10 & 9,21 & 8,81 & 7,40 & 10,35 & $Y=9,07+0,039 x-0,001 x^{2}$ & 0,99 \\
\hline Rend. carcaça quente(\%) & 46,74 & 46,02 & 44,92 & 45,31 & 5,00 & $Y=45,74$ & - \\
\hline Peso carcaça fria $(\mathrm{kg})^{2}$ & 8,99 & 9,12 & 8,62 & 7,32 & 11,23 & $Y=8,98+0,034 x-0,001 x^{2}$ & 0,99 \\
\hline Rend. carcaça fria (\%) & 46,14 & 45,57 & 43,91 & 44,74 & 5,85 & $Y=45,09$ & - \\
\hline Rendimento biológico $(\%)^{2}$ & 63,04 & 61,84 & 60,95 & 64,04 & 4,74 & $Y=63,22-, 200 x+0,004 x^{2}$ & 0,87 \\
\hline Perda resfriamento $(\%)^{2}$ & 1,30 & 1,02 & 0,67 & 1,29 & 51,35 & $Y=1,35-0,047 x+0,001 x^{2}$ & 0,79 \\
\hline Área Longissimus dorsi $\left(\mathrm{cm}^{2}\right)$ & 12,41 & 11,41 & 12,08 & 10,50 & 16,25 & $Y=11,60$ & - \\
\hline Esp. gord. subcutânea (mm) & 0,44 & 0,35 & 0,35 & 0,28 & 35,06 & $Y=0,35$ & - \\
\hline
\end{tabular}

Archivos de zootecnia vol. 61, núm. 233, p. 66. 


\section{CORDEIROS MORADA NOVA ALIMENTADOS COM FENO DE FLOR-DE-SEDA}

$(27,47 ; 27,82 ; 20,27$ e $18,44 \mathrm{~kg})$, respectivamente. Estes autores atribuíram o fenômeno a presença de fatores anti-nutricionais na forragem, conforme relatado por Mello et al. (2001).

Houve influencia da dieta $(\mathrm{p}<0,05)$ sobre as variáveis de conformação da carcaça, comprimento da perna, perímetro da coxa e sobre o índice de compacidade da carcaça. Estes resultados estão de acordo com os obtidos por Pinheiro et al. (2007), trabalhando com cordeiros confinados alimentados com diferentes relações de volumoso: concentrado.

O FFS adicionado na dieta de cordeiros promoveu efeito quadrático $(\mathrm{p}<0,05)$ na característica de comprimento da perna, estimando-se um ponto de máximo em $28,5 \%$. Por outro lado, quando as proporções de FFS nas dietas aumentaram, a conformação, o perímetro da coxa e o índice de compacidade da carcaça diminuíram linearmente, provavelmente em decorrência do menor peso ao abate dos animais nos tratamentos com maiores níveis de inclusão. É importante enfatizar que a conformação da carcaça está estreitamente vinculada à condição corporal e quanto menor o peso vivo ao abate, menos elevada é a conformação. As demais variáveis avaliadas não foram influenciadas $(p>0,05)$ pelos níveis de inclusão de FFS.

Houve efeito quadrático $(\mathrm{p}<0,05)$ para peso do corpo vazio, peso de carcaça quente, peso de carcaça fria, rendimento biológico e perda por resfriamento. Ressalta-se a importância da fibra na determinação do peso do corpo vazio, uma vez que aumentando o teor de FFS na dieta aumenta-se o conteúdo do trato gastrointestinal em função do efeito da fibra na distensão do trato digestório (Bispo et al., 2007). Por outro lado, o conteúdo do trato gastrointestinal decresce quando os níveis de concentrado na dieta se elevam (Medeiros et al., 2008).

Os pesos de carcaça quente e fria apresentaram comportamento quadrático e se ajustaram a pontos máximos de $19,5 \%$ e
$17 \%$ de FFS, respectivamente. Avaliando o efeito de níveis crescentes de fibra em detergente neutro (FDN) na dieta de cordeiros em confinamento, Pires et al. (2006), observaram decréscimo no peso de carcaça quente e peso de carcaça fria. Como neste experimento os teores de FDN também eram crescentes, justifica-se o fenômeno observado.

A partir de $25 \%$ de FFS foi observado um aumento no rendimento biológico. Gonzaga Neto et al. (2006), estudando cordeiros Morada Nova em confinamento, submetidos a dietas com diferentes relações volumoso: concentrado, verificaram que o aumento desta relação promoveu elevação no rendimento biológico. A perda mínima por resfriamento foi observada em $23,5 \%$ de FFS, o que pode estar associada tanto a gordura subcutânea como a localização das carcaças na câmara de refrigeração. Os valores médios da perda por resfriamento nos tratamentos com 0,15 e $45 \%$ de FFS, variaram de 0,67 a 1,30, valores considerados normais de acordo com a literatura consultada (Reis et al., 2001; Araújo Filho et al., 2007). Os rendimentos de carcaça quente e fria, a área do músculo Longissimus dorsi e a espessura de gordura subcutânea não foram influenciadas pelos níveis de FFS na dieta $(p>0,05)$.

Os pesos da meia-carcaça reconstituída, lombo, paleta e costelas diminuíram linearmente em resposta à elevação dos níveis de inclusão de FFS na dieta (tabela III). Os cortes pescoço e filé apresentaram comportamento quadrático $(\mathrm{p}<0,05)$. Não foi observado efeito para o peso da perna $(p>0,05)$. Isto indica que quando o peso da meia carcaça diminui, conseqüentemente, os valores absolutos dos cortes regionais tendem a decrescer.

Com relação aos rendimentos, houve efeito linear positivo $(\mathrm{p}<0,05)$ dos níveis de FFS sobre a percentagem do corte perna, sendo estimado incremento de $0,075 \%$ para cada percentual de FFS na dieta. Observouse efeito linear decrescente nos cortes lombo e costelas. Clementino et al. (2007) observaram efeito linear crescente nos cor- 


\section{SILVA, COSTA, MEDEIROS, AZEVEDO, CARVALHO, MEDEIROS E MADRUGA}

tes lombo e costelas ao estudarem a influencia de diferentes níveis de concentrado na dieta de ovinos mestiços Santa Inês. Portanto, neste estudo, o teor de concentrado da dieta pode ter sido fator determinante no comportamento observado, o que pode ser atribuído ao maior teor de fibra nas dietas com maiores concentrações de feno.

Foi verificado efeito quadrático $(\mathrm{p}<0,05)$ para a percentagem de cabeça, coração,

Tabela III. Composição regional e componentes não constituintes da carcaça de cordeiros Morada Nova em função dos níveis de feno de flor-de-seda (FFS) na dieta. (Regional composition and non carcass components of lambs fed diets containing different levels of silk flower hay (SFH)).

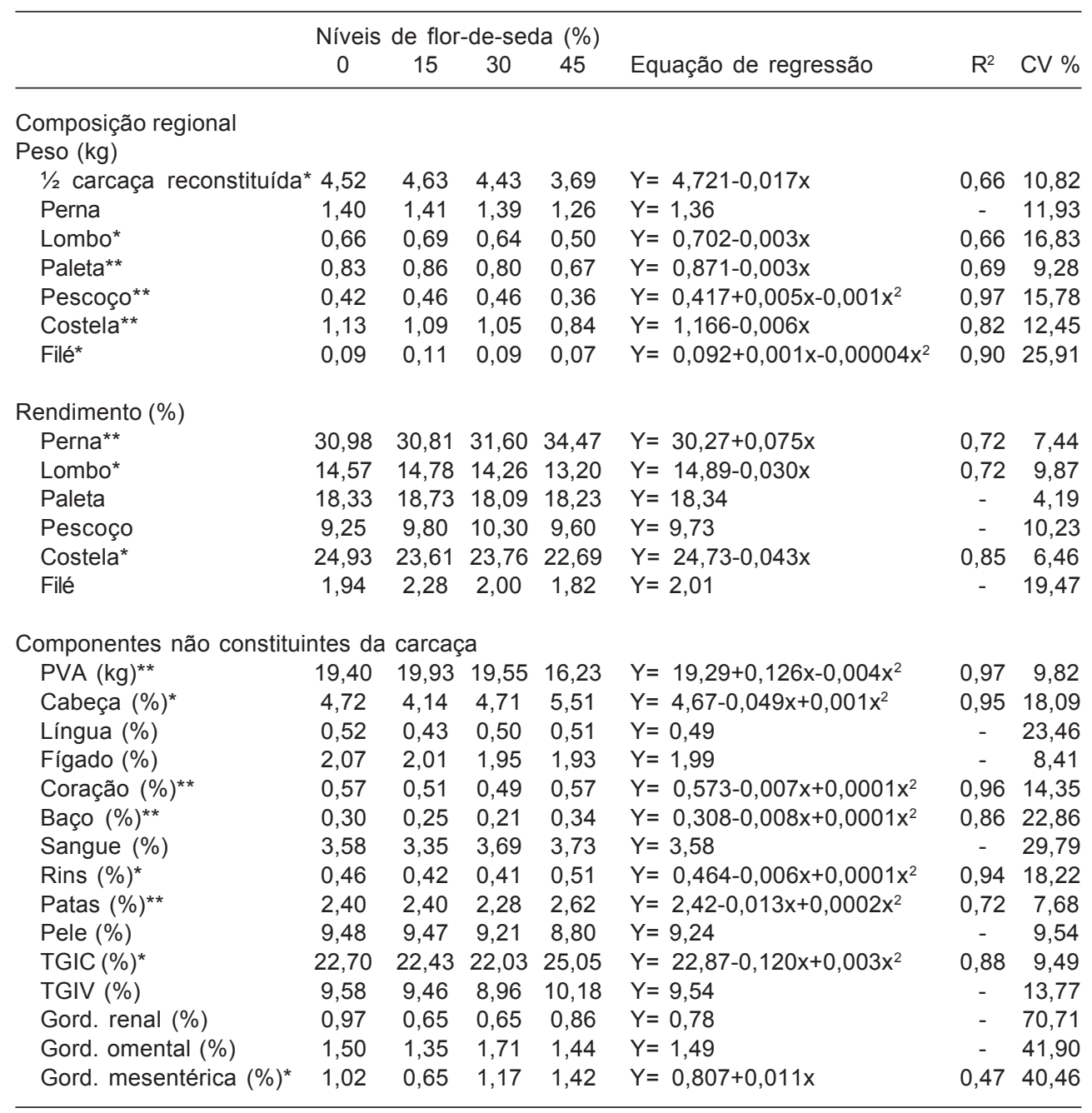

${ }^{*} p<0,05 ;{ }^{* *} p<0,01 ; P V A=$ peso vivo ao abate. TGIC= Trato gastrointestinal cheio; TGIV= Trato gastrointestinal vazio.

Archivos de zootecnia vol. 61, núm. 233, p. 68. 


\section{CORDEIROS MORADA NOVA ALIMENTADOS COM FENO DE FLOR-DE-SEDA}

baço, rins, patas, trato gastrointestinal cheio e gordura mesentérica à medida que se elevaram os níveis de FFS na dieta (tabela III). O nível de $45 \%$ de inclusão proporcionou as maiores médias para estes componentes, podendo está associado ao menor nível de energia metabolizável na dieta, corroborando o raciocínio de Pinheiro et al. (2008), ao relatar que o sistema de terminação de cordeiros em confinamento pode influenciar o peso e o rendimento dos não-componentes da carcaça de cordeiros de diferentes raças e cruzamentos.

Os resultados obtidos neste estudo mostram que a percentagem do trato gastrointestinal cheio se elevou após 20\% de FFS na dieta. Estes resultados estão em concordância aos encontrados por Carvalho et al. (2005), que observaram efeito de níveis de suplementação concentrada em pastagem de Tifton para o peso desta variável. Maiores conteúdos do trato gastrointestinal são observados em animais alimentados com maiores teores de fibra do que concentrado (Osório, 1998). Por outro lado não houve efeito $(\mathrm{p}>0,05)$ dos níveis de inclusão de FFS na percentagem do trato gastrointestinal vazio.

Os níveis de inclusão de FFS promoveram aumento na deposição de gordura mesentérica $(\mathrm{p}<0,05)$, o que reflete o bom estado nutricional dos animais em estudo, bem

\section{BIBLIOGRAFIA}

Alves, K.S., Carvalho, F.F.R., Ferreira, M.A., Véras, A.S.C., Medeiros, A.N., Nascimento, J.F., Nascimento, L.R.S. e Anjos, A.V.A. 2003. Níveis de energia em dietas para ovinos Santa Inês: características de carcaça e constituintes corporais. Rev Bras Zootecn, 32: 1927-1936.

Araújo Filho, J.T., Costa, R.G., Fraga, A.B., Souza, W.H., Gonzaga Neto, S., Batista, A.S.M. e Cunha, M.G.G. 2007. Efeito de dieta e genótipo sobre medidas morfométricas e não constituintes da carcaça de cordeiros deslanados terminados em confinamento. Rev Bras Saud Prod Anim, 8: 394-404. como, a capacidade adaptativa da raça Morada Nova, uma vez que esta deposição de gordura em ovinos tropicais atua como reservas energéticas para serem mobilizadas durante o período de escassez de alimentos

$O$ coração é um órgão prioritário na utilização de nutrientes e mantém sua integridade independentemente do nível de alimentação (Santos et al., 2006), neste estudo, houve efeito da dieta sobre a percentagem deste componente, que apresentou comportamento quadrático e sua derivação ajustou a um ponto de mínimo no nível de $35 \%$ de FFS.

A equação quadrática verificada no valor relativo dos rins, pode ser conseqüência do efeito diurético da Calotropis procera SW relatado por Igbal et al. (2005), em função da alta taxa metabólica relacionada a dieta. As variáveis língua, fígado, sangue, pele, gordura renal e gordura omental não foram influenciados $(\mathrm{p}>0,05)$ pelos níveis de inclusão de FFS.

Em conclusão os resultados evidenciam que o feno de flor-de-seda pode ser usado em até $30 \%$ na alimentação de ovinos em confinamento, podendo com isso manter a produção de carne e, conseqüentemente, do rendimento dos não constituintes da carcaça utilizados na elaboração de pratos típicos regionais preparados com estes componentes.

Bispo, S.V., Ferreira, M.A., Véras, A.S.C., Batista, A.M.V., Pessoa, R.A.S. e Bleuel, M.P. 2007 Palma forrageira em substituição ao feno de capim-elefante. Efeito sobre consumo, digestibilidade e características de fermentação ruminal em ovinos. Rev Bras Zootecn, 36: 19021909.

Carvalho, S., Vergueiro, A., Kieling, R., Teixeira, R.C., Pivato, J., Viero, R. e Cruz, A.N. 2005 Avaliação da suplementação concentrada em pastagem de Tifton-85 sobre os componentes não carcaça de cordeiros. Ciên Rural, 35: 435439

Archivos de zootecnia vol. 61, núm. 233, p. 69. 


\section{SILVA, COSTA, MEDEIROS, AZEVEDO, CARVALHO, MEDEIROS E MADRUGA}

Cezar, M.F. e Souza, W.H. 2007. Carcaças ovinas e caprinas: obtenção, avaliação e classificação. Agropecuária Tropical. Uberaba. 147 pp.

Clementino, R.H., Sousa, W.H., Medeiros, A.N., Cunha, M.G.G., Gonzaga Neto, S., Carvalho, F.F.R. e Cavalcante, M.A.B. 2007. Influência dos níveis de concentrado sobre os cortes comerciais, os constituintes não-carcaça e os componentes da perna de cordeiros confinados. Rev Bras Zootecn, 36: 681-688.

De Boer, H., Dumont, B.L., Pomeroy, R.W. and Weniger, J.H. 1974. Manual on E.A.A.P. reference methods for the assessment of carcass characteristics in cattle. Livest Prod Sci, 1: 151-164.

Gonzaga Neto, S., Silva Sobrinho, A.G., Zeola, N.M.B.L., Marques, C.A.T., Silva, A.M.A., Pereira Filho, J.M. e Ferreira, A.C.D. 2006. Características quantitativas da carcaça de cordeiros deslanados Morada Nova em função da relação volumoso:concentrado na dieta. Rev Bras Zootecn, 35: 1487-1495.

Iqbal, Z., Lateef, M., Jabbar, A., Muhammad, A.G. and Khan, M.N. 2005. Anthelmintic activity of Calotropis procera (Ait.) Ait. F. flowers in sheep. J Ethnopharmacol, 102: 256-261.

Marques, A.V.M.S., Costa, R.G., Silva, A.M.A., Pereira Filho, J.M., Lira Filho, G.E. e Santos, N.M. 2008. Feno de flor de seda (Calotropis procera SW) em dietas de cordeiros Santa Inês: Biometria e rendimento dos componentes não-constituintes da carcaça. Rev Bras Ciênc Agrar, 3: 85-89.

Marques, A.V.M.S., Costa, R.G., Silva, A.M.A., Pereira Filho, J.M., Madruga, M.S. e Lira Filho, G.E. 2007. Rendimento, composição tecidual e musculosidade da carcaça de cordeiros Santa Inês alimentados com diferentes níveis de feno de flor-de-seda na dieta. Rev Bras Zootecn, 36: 610-617.

Medeiros, G.R., Carvalho, F.F.R., Ferreira, M.A., Alves, K.S., Matos, C.W., Saraiva, T.A. e Nascimento, J.F. 2008. Efeito dos níveis de concentrado sobre os componentes nãocarcaça de ovinos Morada Nova em confinamento. Rev Bras Zootecn, 37: 1063-1071.

Mello, M.M., Vaz, F.A., Gonçalves, L.C. e Saturnino, H.M. 2001. Estudo fitoquimico da Calotropis procera Ait., sua utilizacao na alimentacao de caprinos: Efeitos clínicos e bioquímicos sericos. Rev Bras Saud Prod Anim, 2: 15-20.

NRC. 1985. National Research Council. Nutrient requirements of sheep. National Academy Press. Washington, D.C. 99 pp.

Osório, J.C.S., Osório, M.T.M. e Jardim, P.O.C 1998. Métodos para avaliação de carne ovina in vivo na carcaça e na carne. Universidade Federal de Pelotas. Ed. Universitária. Pelotas. $107 \mathrm{pp}$.

Pinheiro, R.S.B., Silva Sobrinho, A.G., Marques, C.A.T. e Yamamoto, S.M. 2007. Biometria in vivo e da carcaça de cordeiros confinados. Arch Zootec, 56: 955-958.

Pinheiro, R.S.B., Silva Sobrinho, A.G., Gonzaga Neto, S., Yamamoto, S.M., Mourão, R.C., Homem, A.C. e Santos, V.C. 2008. Rendimento dos nãocomponentes da carcaça de cordeiros de diferentes genótipos. Arch Zootec, 57: 71-74.

Pires, C.C., Galvani, D.B., Carvalho, S., Cardoso, A.R. e Gasperim, B.D. 2006. Características da carcaça de cordeiros alimentados com dietas contendo diferentes níveis de fibra em detergente neutro. Rev Bras Zootecn, 35: 20582065.

Reis, W., Jobim, C.C., Macedo, F.A.F., Martins, E.N. e Cecato, U. 2001. Características da carcaça de cordeiros alimentados com dietas contendo grão de milho conservados em diferentes formas. Rev Bras Zootecn, 30: 1308-1315.

Santos, N.M., Costa, R.G., Medeiros, A.N., Madruga, M.S. e Gonzaga Neto, S. 2006. Caracterização dos cortes comestíveis não constituintes da carcaça de caprinos e ovinos. Agropec Tecn, 26: 97-108.

Santos, N.M., Costa, R.G., Madruga, M.S., Medeiros, A.N., Albuquerque, C.L.C. and Queiroga, R.C.R.E. 2008. Constitution and composition chemistry of the precooked goatlike buchada produced in the State of Paraíba, Brazil. Braz Arch Biol Techn, 51: 593-598.

SAS. 1996. SAS User's Guide. Version 6.11. $4^{\text {th }}$ ed. SAS Institute Inc. Cary. v. 2. 842 pp.

Silva, N.V., Fraga, A.B., Araújo Filho, J.T. Cavalcante Neto, C.C., Silva, F.L., Costa, P.P.S. e Lira Júnior, W.B. 2007. Caracterização morfométrica de ovinos deslanados Cabugí e Morada Nova. Rev Cient Prod Anim, 9: 33-37.

Archivos de zootecnia vol. 61, núm. 233, p. 70. 